\title{
Simulation of HVDC control and transmission lines protection based on PSCAD/EMTDC
}

\author{
ChaoQun Sheng ${ }^{1, a}$ \\ ${ }^{1}$ North China Electric Power University, Hebei 071000, China; \\ a327417065@qq.com
}

Keywords: HVDC bus differential protection, PSCAD simulation

\begin{abstract}
HVDC control and protection system was introduced in this paper. The control generally use fixed-current controlin rectifier and fixed arc angle controlin inverter. The short circuit intransmission line is one of the most common faults in HVDC, so this paper choose HVDC bus differential protection as research object,introduce the mechanism of bus differential protection and use PSCAD software for simulation. This paper is mainly about thebus differential protection and auto-reclosing after the breaker action.

In the simulation process, we encountered the following three questions:

1. How to convert current difference to logic signal;

2. How tocontrolthe action of circuit breaker with the control signal changing;

3. Why circuit breakers fail to function.

And we put forward concrete solutions towards the above three questions.
\end{abstract}

\section{Introduction}

\subsection{The significance of study on HVDC [1]}

China's natural resources and characteristics of energy and load distribution make long distance and large-capacity transmission become inevitable. To lessenthe transmission line loss and save precious land resources, we need aneconomical and efficient way of transmission.Precisely, HVDC technology meet this requirement. At present, China has become the country with the world's largestDC transmission capacity, most DC projects, and the highest levels of DC voltage. The next 20 years, with the acceleration of grid construction and the development and utilization of new energy resources, HVDC technology in China will be further strengthened. HVDC has become the most attractive and most notably development direction in present power industry.

Development of HVDC technology significantly promotethe development of power system, mainly manifested in the following areas:

Expand applicativeadvantage in long-distance transmission and networking;

Promote the functioning of the electricity market;

Promote the development and utilization of renewable energy.

\subsection{The structure of HVDC [2]}

Basic principles of HVDC is shown in Figure 2-1. HVDC system includes two converter stations CS1 and CS2 and DC lines. Converter in converter station can achieve the interconversion between AC and DC.

Converter consists of one or more converter bridge in series or parallel. Now DC transmission system use three-phase bridge converter circuit, each bridge has 6 arms. Due to the unidirectional flux of the arm can be controlled, it is also known as valves. Arms consist of the mercury arc valves or electronic switching devices (such as thyristor) in series. Modern HVDC has basically eliminated the converter made by mercury arc valve. 


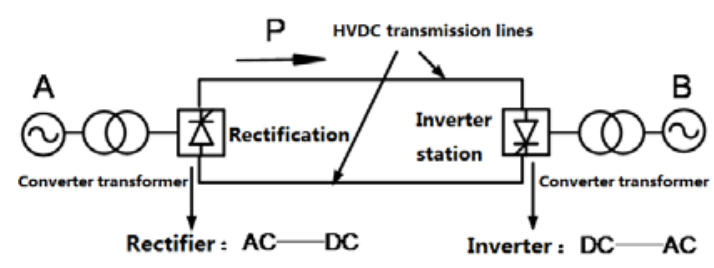

Fig. 2-1 the basic principle of HVDC

WhenAC powersystem I transmit power to the AC powersystem II, converter station CS1 will transformthe three-phase AC from system I into the DC, then send power via converter station in HVDC transmission lines to converter station CS2, and CS2 convert DC to AC. In general, AC-DC is calledrectifier, while DC-AC is called inverter. Thus CS1, also known as rectification, and CS2 called inverter station.

We set CS1 DC input voltage $V_{d 1}$ and CS2 DC output voltage $V_{d 2}$, then the DC line current is:

$$
I_{d}=\left(V_{d 1}-V_{d 2}\right) / R(1-1)
$$

Where $R$ is the resistance of the DC line. It should be noted that DC line only transfer active power without reactive power. The $P_{d 1}$ and $P_{d 2}$ respectivelyimply the supplying power in CS1 and the absorbing power in CS2.

$$
P_{d 1}=V_{d 1} I_{d} P_{d 2}=V_{d 2} I_{d}
$$

The difference between the two values is the loss of DC line:

$$
\Delta P=I_{d}\left(V_{d 1}-V_{d 2}\right)(1-3)
$$

\subsection{The control system of HVDC}

The operating characteristics of HVDC system consists of DC current regulator, DC voltage regulator and arc angle regulator three basic controller. Through the coordination among each segment of the control regulation,under different operating conditions, it makes the whole DC system operate steadily. In the pole control system, through configuring different parameters in the rectifier and inverter,it makes the rectifier and inverter work by different regulator during actual operation, thus getting the ideal Volt-Ampere characteristic curve.

The control input variables of DC transmission control system except the rectifier current set value signal $I_{d e s}$ from main control layer, also haverectifier DC current $I_{d-r e c}$, and inverter DC current $I_{d-i n v}$, and inverter DC voltage $U_{d-i n v}$, and inverterextinction angle $\gamma_{-i n v}$ measured from DC system. The output variables of DC transmission control system are only 2, respectively for rectifier firing angle $\alpha_{r e c}$ and inverterfiring angle $\alpha_{i n v}$ 。

\section{The protection of HVDC [3]}

\subsection{The fault on DC line}

Short circuit fault is one of the most common faults in HVDC, lightning, dirty, corrosion and other factors will lower the insulation level of the line,arcing faultoccurs when serious, resulting in DC line short circuit fault. The phenomenon is when occurring short circuit fault in DC transmission line: in rectifier, DC voltage fall and DC current rise while in inverter, DC voltage and DC current decline.

\subsection{The DC bus protection}

DC bus area is the link between overhead wiring, grounding wire and the converter. To accurately distinguish between its external and internal fault and ensure the selectivity of relay protection, differential protection principle is generally used to protect it. Common DC bus protection include:

a) HVDC bus differential protection,used to protect HVDC bus to ground and neutral DC bus fault;

b)Neutral DC bus differential protection, used to protect the neutral bus to ground short-circuit fault;

c) DC backup differential protection, used to protect both the DC bus area fault and the converter area ground fault, commonly used as a general backup protection for the ground fault in two areas. The detailed description of HVDC bus protection is showed below [4]. 


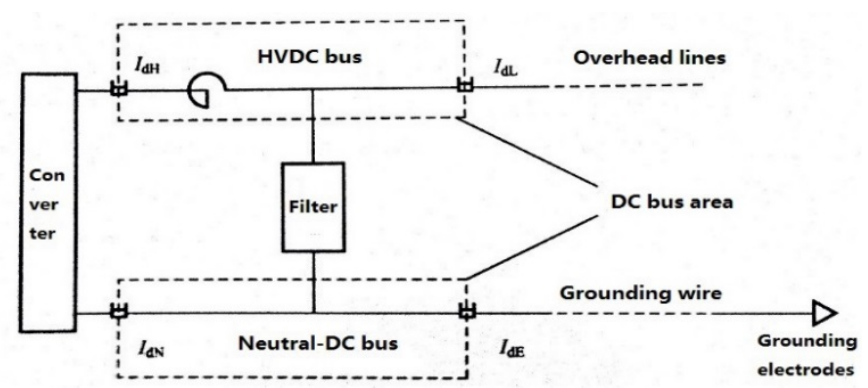

Fig. 5-1 the structure of DC bus area

High voltage DC bus differential protection tests high voltage terminal current $I_{d H}$ and overhead lines current $I_{d L}$ at inverter DC export. Under normal conditions, as well as HVDC bus fault outside the DC bus area, the both currents are essentially equal. When occurring to ground or to neutral short circuitinside, there is a big difference between $I_{d L}$ and $I_{d H}$.

The basic criteria for HVDC bus differential protection is showed below: $\left|I_{d H}-I_{d L}\right|>I_{\text {set }}(2-1)$

Where $I_{\text {set }}$ is the start current setting value.The character considering the minimum action current and the braking characteristics is:

$I_{r}>\max \left(I_{\text {setmin }}, K_{\text {set }} I_{\text {res }}\right)(2-2)$

Where $I_{r}=\left|I_{d H}-I_{d L}\right|$ is action current; $I_{\text {res }}=\max \left(I_{d H}, I_{d L}\right)$ is braking current; $K_{\text {set }}$ is ratio braking coefficient, which need to be considered to avoid inverter area, the current through the filter when the most serious fault on DC lines and maximum measuring through current error of the two current transformers, it generally takes about $0.2 ; I_{\text {setmin }}$ isthe minimum action current, considering transformer stray noise and the unbalance value between $I_{d L}$ and $I_{d H}$ caused by ground fault inside filter, soit generally takes about 0.3 .

\subsection{Automatic reclosing}

For non-permanent faults, automatic reclosing is undoubtedly an important means to improve the quality of power supply, but differs from the AC system protection, before closing, it needs to be confirmed whether non-permanent faults occurin the line, so adding the line detection. DC bus primary protection, circuit detectionand automatic reclosing ensure the requirements for DC protection: rapidity, selectivity and reliability.The simulation only study on transient fault, so we omit the line detection device.

\section{Simulation}

\subsection{The model}

International Council on Large Electric systems(CIGRE) standard DC transmissiontesting systems (CIGRE HVDC Benchmark Model) is used for the study of HVDC control system. It is easy to use simulation program or emulator to study on different DC main circuit control devices and comparative performance of control strategies in similar model, its DC main circuit structure is fairly simple, but its operational conditions are difficult [5].

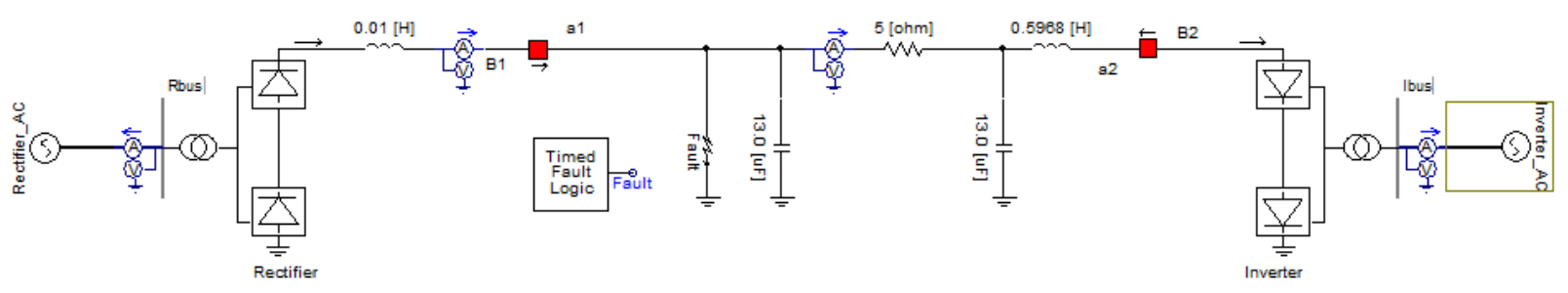

Fig. 3-1 the simulation circuit of DC bus fault and protection

In the simulation circuits, we set short circuit fault occurs at time point $0.2 \mathrm{~s}$ and the fault lasting time is $0.1 \mathrm{~s}$. 


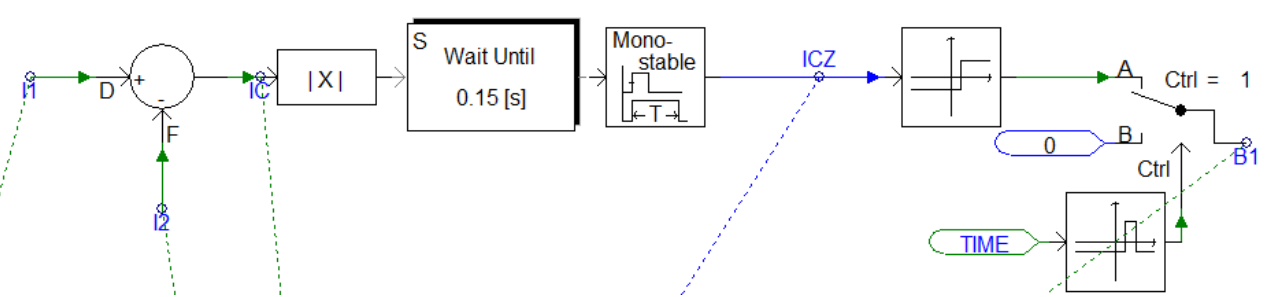

Fig. 3-2 the protection control system of HVDC bus

In the control system showed in the figure 3-2. We let the two measured signal go throughthe element: "Summing/Difference Junction", “Absolute Value”, "Wait for an Event", "MonostableMultivibrator", "Single Input Comparator", “Two Input Selector” in sequence. We let time signal firstly go through "Range Comparator" and then to the ctrl terminal of "Two Input Selector”.

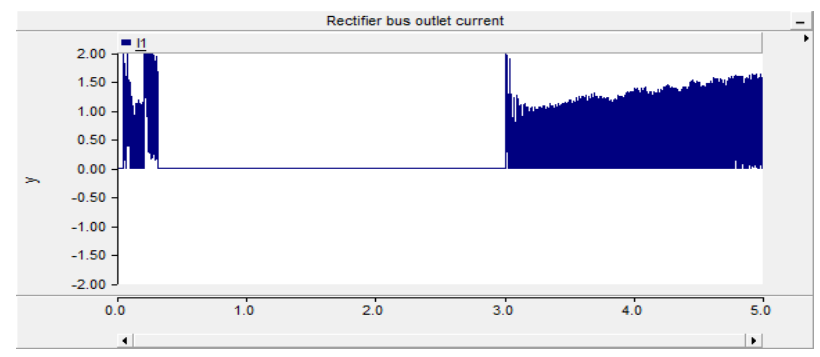

Fig. 3-3 rectifier bus outlet current

From the figure 3-3, we can clearly see that breaker acts after fault occurring, the current goes to zero. After some time, at about time point 3.0s, automatic reclosing acts, the current regain.

\section{Summary}

Throughout the whole implementation process, we encountered the following problem:

a) Circuit-breaker opening and closing control is achieved through the signal "ON=1" or "OFF $=0$ " to the node, but the differential protection principle is two current differencewhich is real value, we need to convert real numerical values to logical value 0 or 1 . By introducing a single input comparator has perfectly solve this problem.

b) The breaker is controlled by the current difference measured by values on both sides of power meters. It means working normally when the difference is small andopen the breaker when the difference is too great. But after opening the breaker, the current on both sides are 0 , the difference is also 0.This immediately meets the circuit breaker closing conditions, causing circuit breakers sometimes openedor closed. Monostable multi-frequency oscillator can solve this problem.

\section{Reference}

[1] Li Jiaman. DC protection response and exit timing characteristics of AC fault [d]. South China University of technology, 2015.

[2] King week. Fault analysis and protection for high voltage AC and DC power systems [d]. Zhejiang University, 2014.

[3] Xing Luhua. Study on high voltage direct current transmission line protection and fault location principle [d]. Shandong University, 2014.

[4] Xu min. DC circuit analysis method of relay protection and research on protection principle [d]. South China University of technology, 2014.

[5] Qi Yan. Fault analysis and protection scheme of HVDC transmission system [d]. East China Jiaotong University, 2012. 